\title{
The Effectiveness of Relapse Prevention Intervention on the Ability of Patients and Their Families to Prevent Psychotic symptoms of Relapse among Patients with Schizophrenia: Systematic Literature Review
}

\author{
Mohammad A. Abu Sabra' ${ }^{1}$, Ayman M. Hamdan-Mansour ${ }^{2}$ \\ ${ }^{1}$ PhD Candidate, School of Nursing, Al-Zaytoonah University of Jordan (ZUJ), Amman, Jordan, ${ }^{2}$ Professor, \\ School of Nursing, the University of Jordan, Amman, Jordan
}

\begin{abstract}
Background: Most patients with schizophrenia have family support. The role of a family as a care partner has the capability of expanding the patient's recovery and solving the family's need for awareness, encouragement, and improves skills. Objective: Assessing the effectiveness of relapse prevention intervention on the ability of patients and their families that are reported to prevent psychotic symptoms of relapse among patients with schizophrenia. Method: A systematic review was conducted following Preferred Reporting Items for Systematic Review and Meta-Analysis (PRISMA) guidelines for the studies published between 2010 and 2020. Results: Twelve retrieved studies were shown the relapse prevention intervention increases the ability of patients and their families to minimize and prevent psychotic symptoms. The relapse prevention interventions in this review including; optimal case management, multifamily group psycho-education, integrated psychological intervention, psychosocial treatment, Information Technology Aided Schizophrenia Relapse Prevention, family-focused therapy, and Family psycho-education programs. Conclusion: Various relapse prevention interventions have been reported to reduce the risk of relapse rate, prevent psychotic symptoms, and sustain remission by incorporating and emphasize the importance of effective cooperation families with patients with psychotic relapse.
\end{abstract}

Keywords: Early warning sign, family intervention, psychosocial intervention, psychotic relapse, relapse prevention intervention, and schizophrenia.

\section{Introduction}

Schizophrenia affects 20 million people worldwide and is a chronic and severe mental disorder ${ }^{1}$. It is one of the most common and troubling signs of mental illness that leads to psychosocial difficulties that are destructive in life, as well as a major burden on patients, families, social, and clinical is correlated with psychotic relapse outcomes ${ }^{2-3}$. Schizophrenia is a mental illness that involves, first of all, positive signs like; delusions, interruption of the thinking process, and hallucinations.

Corresponding author:

Hamdan-Mansour:

a.mansour@ju.edu.jo.
Secondly, negative symptoms like; diminished emotional expression, social relationship problems, and motor weakness. Last, cognitive symptoms like; misunderstanding, memory loss, impaired judgment ${ }^{4}$.

Psychotic relapse is a reoccurrence episode and devastating consequences in schizophrenia patients, including worsening symptoms and decreased quality of life ${ }^{5}$. Furthermore, there is always a span of 1-4 weeks with signs of early warnings that precede relapse ${ }^{6}$. Therefore, appropriate steps to promote early intervention should be taken when early warning signs (prodromal symptoms) of psychosis occur and are reported ${ }^{7}$. Consequently, relapse prevention intervention is a strategy to minimize the risk and intensity of relapse following the cessation or decrease of problematic habits 
and is a step towards sustained healing and remission; one of the most important measures to be taken to ensure that a client lives a stable recovery life ${ }^{8}$.

In Jordan, most studies are focusing on general sociocultural factors while addressing mental health rather than interventional ones ${ }^{9-10}$. Unfortunately, the very few studies examined effectiveness of CBT has focused on university students ${ }^{11}$ and abused women ${ }^{12}$, while studies examined relapse in general focused on individuals with substance use problems ${ }^{13}$. Moreover, patients with schizophrenia have been addressed from general perspective such quality of life ${ }^{14}$. However, investigating relapse intervention have not been addressed before in Jordan and neighboring countries. Therefore, relapse prevention intervention need to be investigated for its promising benefits ${ }^{15-16}$.

\section{Objective}

The aim of this review was to assess the effectiveness of relapse prevention intervention on the ability of patients and their families to prevent psychotic symptoms of relapse among patients with schizophrenia.

\section{Methods}

\section{Searching Strategy}

The literature searching period covered published papers from 2010 until 2020. The search of the appropriate databases was carried out to evaluate the current evidence for the proposed rsearch aims of this study. In order to begin a literature search, it was important to suggest definitive search terms to support the overall literature search limit. The terms used included; relapse prevention intervention, psychotic relapse, schizophrenia, psychosocial intervention, early warning sign ( prodromal symptoms), and family intervention.

\section{Information Sources}

An extensive and comprehensive review of the literature by conducted a various database that has been actively used including; Medline, EBSCO, PubMed, Google Scholar, and Wiley Online Library databases, CINAHL Plus, and ProQuest databases.

\section{Eligibility criteria}

The requirements for inclusion were: 1) English language publications; 2) the studies that were reviewed focused on relapse prevention intervention that prevent psychotic symptoms of relapse; 3) design of the study, qualitative, quantitative, and mixed methods; 4) published between 2010 - 2020. on the other hand, The articles were excluded if they were: (1) publication are reviews, editorials, letters, and reports; 2) not relevant to the terminology and understanding of relapse prevention intervention; 3) studies that had been published in different languages other than English; 4) studies published prior to the year 2010. In order to exclude the redundant and duplicated articles, filtration was performed on all articles.

\section{Study selection}

Initially, the combination of these keywords, as mentioned above was searched using the electronic databases selected. The following results were produced by this search: relapse prevention intervention (173 records), psychotic relapse (109 records), schizophrenia (30,680 records), psychosocial intervention (4,704 records), early warning sign (prodromal symptoms) (1,934 records), and family intervention(7,643). Then, 49 articles came back looking for the combination of these keywords using the same electronic databases as well as additional records identified through other sources are $(n=17)$ articles. then removing duplicates resulted in $(n=$ 14) articles. After that, Records excluded; Title or abstract indicated that the study is irrelevant to relapse prevention intervention on the ability of patients and their families to prevent psychotic symptoms of relapse, or not written in English, or not within specified timeframe $(n=16)$ of articles. only, after full-text articles excluded; were not eligible according to eligibility criteria: the papers were not research (publications are reviews, editorials, letters, and reports), or did not address nursing interventions (n $=24)$. Finally, the total articles included in this study ( $\mathrm{n}=$ 12) of article.

\section{Data collection process}

All team members revised every article to recognize the relevant points. Discussions between team members have overcome the discrepancy about the eligibility of 
studies. The researchers discussed their findings and patterns, and inferences were identified from those trends.

\section{Data items}

The researchers conducted an in-depth evaluation of the findings of the included study that were outlined in a review matrix.

\section{Risk of bias in individual studies}

The researchers analyzed the probability of bias separately in all studies. Using the Critical Appraisal Skills Programme (CASP) Qualitative Research Checklist was evaluated ${ }^{17}$. While the quantitative studies were evaluated using the Quality Evaluation Tool (QAT) checklist derived from the Successful Public Health Practice Project (EPHPP) $)^{18}$.

\section{Synthesis of Results}

Reviewers completed the synthesized and analyzed data; any gaps between them were set by consensus.
Synthesis of the evidence was made, including continuity of findings and key findings, in addition to tables, across studies.

\section{Result}

\section{Study Characteristics}

The 12 articles reviewed for this study were published between 2011 and 2019. All of them include relapse prevention intervention method (strategies) on the ability of patients and their families to prevent psychotic symptoms of relapse among patients with schizophrenia. 11 of 12 articles were quantitative studies and the only one article is a qualitative study. The majority of the studies were published in 2011, 2013, 2014, 2015, and 2016 with a percent of $(2 / 12,15.66 \%)$, two articles for each year, and there was just one article was published in 2012 , and 2019 with a percent of $(1 / 12,8.3 \%)$ for each year. A summary of studies characteristics is shown in table 1. Show the number of sample size, study design, and country of study with percentage.

Table 1: Characteristics of the study included in the review $(\mathrm{N}=12)$

\begin{tabular}{|c|c|c|}
\hline & N (\%) & References (no.) \\
\hline \multicolumn{3}{|c|}{ Sample size } \\
\hline$<50$ & $3(25 \%)$ & $\begin{array}{l}\text { Breitborde et al.,2011; Komatsu et al., 2013; Eisner et } \\
\text { al., } 2014\end{array}$ \\
\hline $50-200$ & $5(41.7 \%)$ & $\begin{array}{l}\text { Sungur et al.,2011; Bechdolf et al.,2012; Weisman de } \\
\text { Mamani et al., 2014; Mayoral et al., 2015; Marvin et al., } \\
2016\end{array}$ \\
\hline $200-400$ & $3(25 \%)$ & $\begin{array}{l}\text { Gleeson et al., 2013; Ran et al., 2015; Fikreyesus et al., } \\
\qquad 2016\end{array}$ \\
\hline$>400$ & $1(8.3 \%)$ & Niksalehi et al., 2019 \\
\hline \multicolumn{3}{|c|}{ Study design } \\
\hline Qualitative (RCT design) & $9(75 \%)$ & $\begin{array}{l}\text { Sungur et al.,2011; Breitborde et al.,2011; Bechdolf et } \\
\text { al.,2012; Gleeson et al., 2013; Komatsu et al., 2013; } \\
\text { Weisman de Mamani et al., 2014; Mayoral et al., 2015; } \\
\text { Ran et al., 2015; Marvin et al., } 2016\end{array}$ \\
\hline $\begin{array}{l}\text { Quantitative (quasi-experimental } \\
\text { design) }\end{array}$ & $1(8.33 \%)$ & Niksalehi et al., 2019 \\
\hline $\begin{array}{l}\text { Quantitative ( a cross-sectional } \\
\text { study) }\end{array}$ & $1(8.33 \%)$ & Fikreyesus et al., 2016 \\
\hline
\end{tabular}


Cont.... Table 1: Characteristics of the study included in the review $(\mathrm{N}=12)$

\begin{tabular}{|c|c|c|}
\hline $\begin{array}{c}\text { Qualitative (A thematic approach } \\
\text { in-depth interviews) }\end{array}$ & $1(8.33 \%)$ & Eisner et al., 2014 \\
\hline \multicolumn{2}{|c|}{ Country } \\
\hline Turkey & $1(8.33 \%)$ & Sungur et al.,2011; \\
\hline USA & $3(25 \%)$ & $\begin{array}{c}\text { Breitborde et al.,2011; Weisman de Mamani et al., 2014; } \\
\text { Marvin et al., 2016 }\end{array}$ \\
\hline UK & $1(8.33 \%)$ & Eisner et al., 2014 \\
\hline German & $1(8.33 \%)$ & Bechdolf et al.,2012 \\
\hline Australia & $1(8.33 \%)$ & Gleeson et al., 2013 \\
\hline Japan & $1(8.33 \%)$ & Komatsu et al., 2013 \\
\hline Spain & $1(8.33 \%)$ & Mayoral et al., 2015 \\
\hline Chinese & $1(8.33 \%)$ & Ran et al., 2015 \\
\hline Iran & $1(8.33 \%)$ & Fiksalehi et al., 2019 \\
\hline Ethiopia & $1(8.33 \%)$ & \\
\hline & & \\
\hline
\end{tabular}

Risk of Bias within Studies

The quality of the chosen quantitative studies was evaluated by using the Effective Public Health Practice Project (EPHPP) Quality Assessment Tool ${ }^{18}$. In general; study reports show 9 studies out of eleven with good enough quality ratings. Only two studies out of eleven were rated between good and fair on controlling for the selection bias. Moreover, the studies that were included in the study notify very important outcomes. In order to assess the quality of qualitative research, the Critical Appraisal Skills Program (CASP) Qualitative Research Checklist was evaluated ${ }^{19}$. The research report reveals, in total, one study out of one with good quality ratings. Shown in Table 2

Table 2: Risk of Bias within Studies

Effective Public Health Practice Project (EPHPP) Quality Assessment Tool for quantitative studies (n=11)

\begin{tabular}{|c|c|c|c|c|c|c|c|c|c|c|c|c|}
\hline $\begin{array}{l}\text { Number } \\
\text { of items }\end{array}$ & Items & 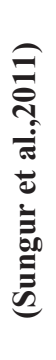 & 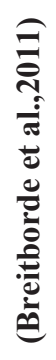 & 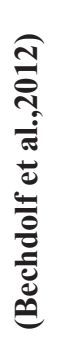 & 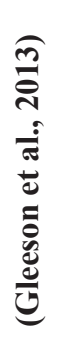 & 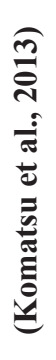 & 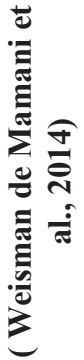 & 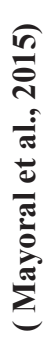 & 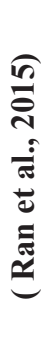 & 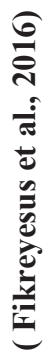 & 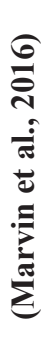 & 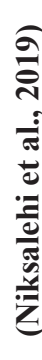 \\
\hline 1. & Selection Bias & 1 & 1 & 1 & 1 & 1 & 1 & 1 & 1 & 1 & 1 & 1 \\
\hline 2. & Study Design & 1 & 1 & 1 & 1 & 1 & 1 & 1 & 1 & 2 & 1 & 2 \\
\hline
\end{tabular}


Cont... Table 2: Risk of Bias within Studies

\begin{tabular}{|l|c|c|c|c|c|c|c|c|c|c|c|c|}
\hline 3. & Control for confounders & 2 & 2 & 1 & 1 & 1 & 1 & 1 & 1 & 2 & 1 & 2 \\
\hline 4. & Blinding & 1 & 1 & 1 & 1 & 1 & 1 & 1 & 1 & 2 & 1 & 1 \\
\hline 5. & $\begin{array}{c}\text { Data Collection Methods } \\
\text { Withdrawals and Drop- } \\
\text { Outs }\end{array}$ & 1 & 1 & 1 & 1 & 1 & 1 & 1 & 1 & 1 & 1 & 1 \\
\hline 6. & \begin{tabular}{c} 
Out \\
\hline
\end{tabular}
\end{tabular}

Key: $1=$ strong, $2=$ moderate, $3=$ weak, NA: not applicable

\section{Results of Individual Studies}

Identification of commonly relapse prevention intervention on ability of patients and their families to prevent psychotic symptoms of relapse.

Research validates a range of relapse prevention interventions for early psychotic symptoms. Several trials have been performed on new combination between pharmacological (medication treatment) and non-pharmacological treatments (psychosocial intervention) that are effective for people with schizophrenia, and housing facilitation supported by assisted living and supported employment are productive coping mechanisms ${ }^{1}$. The twelve reviewed studies were conducted to describe the most commonly the effectiveness of relapse prevention intervention on the ability of patients and their families to prevent psychotic symptoms of relapse among patients with schizophrenia. All of these studies targeted nurses, clinicians, patients, and their families in inpatients and outpatients' schizophrenia.

The first study was performed at Psychiatric hospital in Ankara (turkey). For one hundred patients diagnosed with schizophrenia, it uses either routine case management (RCM) or optimal case management (OCM) randomly assigned to 24 months. During the first three months, both patients with schizophrenia and their families attended 120-minute training sessions at the patient's home every 2 weeks; 45 -minute weekly sessions were held at the outpatient setting for the rest of the 24-month study duration to support patients and their family deal with early psychotic symptoms ${ }^{20}$.
The second study was conducted at the Intervention Centers for Early Psychosis. Forty first-episode psychosis patients who enroll simultaneously in multifamily group psycho-education (MFG) and cognitive remediation may be less likely to relapse and enhance cognitive functioning ${ }^{2}$. The third study was carried out in German at the center of intervention and early detection. Integrated psychological intervention (IPI) Over a 12-month timeframe, treatments were administered to 128 outpatients, then follow-up was carried out for up to 24 months in an early initial prodromal state (EIPS) to a psychotic reduction $^{21}$.

The fourth study was carried out in Australia at Prevention and intervention Centre. First-episode psychosis (FEP) programs have evaluated the efficacy of a new seven-month psychosocial therapy intended to minimize the second episode of psychosis and minimize hospital readmissions. Forty patients with first-episode psychosis were randomly chosen to receive specialist FEP care. Baseline, 7, 12, 18, 24 and 30 months follow-up patients are measured ${ }^{22}$. The fifth study was performed at four hospital institutions in Japan. The Information Technology Aided Relapse Prevention Program for Schizophrenia (ITAREPS) and control group have been randomized to 45 outpatients with schizophrenia and have been monitored for 12 months. It has been implemented as a relapse prevention program ${ }^{23}$.

Sixthly, while Focusing on psycho-educational family intervention program and family-focused therapy. Five studies emphasized the impact, efficacy, and effectiveness of these intervention programs on the ability of patients with their families to prevent and minimize psychotic relapse. The first study explored that family interventions can help schizophrenia patients by created a culturally aware, family-focused treatment 
for schizophrenia patients (CIT-S). Sixty-nine families were randomized to 15 CIT-S sessions or to a 3-session screening condition for psycho-education (PSY-ED). Conducted on Miami's at different institution (hospital and mental health center $)^{24}$. The second study was executed in Spain at a clinic for mental health. It is recognized to be one of the most valuable psychosocial interventions for patients with schizophrenia and family psycho-educational programs. This randomized 88 families into two categories. 12 months of psychoeducational intervention were obtained by the family intervention group and the other group sought regular routine care. follow up were performed at baseline, 12 months, and 18 months $^{18}$.

The third study was conducted in China. In a facility for mental wellbeing. 326 schizophrenia patients are randomized into three classes and psycho-educational family intervention is extended to schizophrenia patients to examine the 14-year influence of psycho-educational family intervention on schizophrenia patients ${ }^{23}$. The fourth study was performed in a mental health facility in North America.103 patents for positive psychosis. Families are interested in two randomized psychosocial approaches: family-focused therapy for patients at psychiatric higher possibility for psychosis (FFT-CHR) (18 sessions over 6 months) and enhancement of care (EC) (three sessions over 1 month). It is used to support family members during psycho-education to determine sources of symptoms and useful coping strategies ${ }^{26}$. Similarly, the last study performed at a psychiatric hospital in Iran clarifies that family psycho-education is used as an intervention for patients with a mental illness by strengthening the awareness of the situation, risk factors, and recovery strategies for reducing relapse by the caregiver and families. 4049 participants were committed to a one-session individual family psychoeducational intervention directly after discharge ${ }^{27}$..

The seventh study was done in England at a mental health clinic. A thematic approach was used in a qualitative study to analyze data from in-depth interviews with psychosis patients (20-25 participants) and to improved understand the early relapse phase, the willingness of patients and their families to recognize early signs and any possible barriers and facilitators to early sign interviewing ${ }^{28}$. The final study was performed in Southwestern Ethiopia at a special hospital. Data were obtained in this cross-sectional study using an interviewer-administered questionnaire for 386 psychotic relapse patients and offering treatment, psycho-education, and psychosocial assistance to help patients improve compliance with treatment and reduce psychotic relapse ${ }^{29}$.

Evaluation of the effect of used relapse prevention intervention on ability of patients and their families to prevent psychotic symptoms of relapse

Overall, the twelve studies were conducted to appraise the effectiveness of relapse prevention intervention on the ability of patients and their families to prevent psychotic symptoms of relapse among patients with schizophrenia. All of the studies showed there is a strong relationship between effective uses of relapse prevention intervention strategies and the ability of patients and their families to dealing with psychotic symptoms to prevent psychotic relapse.

First of all, the result of the study conducted by Sungur et al. ${ }^{30}$ showed optimal case management (OCM) is statistically and clinically significant benefits. as well as OCM is focusing and helping patients and their families by training theme different relapse prevention intervention strategies to relieve stress that is lead to psychotic relapse such as social skills, work support, cognitive-behavioral interventions for psychotic symptoms, and anger control. Secondly, Breitborde et al ${ }^{31}$ revealed two key findings; patients with firstepisode psychosis and their families can be hired to participate, and the main outcome of this research is a relief relapse of psychotic symptoms. The secondary results for enhancing the capacity of psychotic patients and their families to understand and cope with psychotic symptoms. Thirdly, a study undertaken by Bechdolf et al ${ }^{31}$ assures that at 12, 24-month follow-up, integrated psychological intervention was better to supportive treatment in preventing psychosis relapse and finding patients to be safe, well-accepted, and tolerated.

Fourthly, a study revealed by Gleeson et al ${ }^{22}$ showed that the relapse rate in the treatment state was significantly lower at 12 -month follow-up relative to 
advanced treatment alone. The reported relapse rate of around $35 \%$ in excess of 2-year follow-up in specialist FEP care is beneficial compared to previously reported relapse rates of $55 \%-70 \%$. Fifthly, a study held out by Komatsu et $\mathrm{al}^{23}$ ensures that ITAREPS is extremely effective. However, the efficacy was influenced by user compliance to the program protocol, the accurate efficiency and function of ITAREPS was partly unknown. For all patients and their families to identify the early warning signs, ITAREPS uses EWSQ (early warning sign questionnaires) via weekly phone calls. The willingness of patients and their families to track and stop psychotic symptoms is also effective.

Sixthly, the finding and evaluation of five studies that are focusing on psycho-educational family intervention programs and family-focused therapy. Five studies show the significant and positive effects of these intervention programs on the ability of patients with their families to prevent and minimize psychotic relapse as the following: The first study performed by Weisman de Mamani et al ${ }^{24}$ found that CIT-S at the end of treatment had significantly fewer debilitating health issues than patients referred to PSY-ED. The patient's race and the racial match of the patient-therapist did not contribute to the success of treatment or the satisfaction of intervention. The second research conducted by Mayoral et al ${ }^{32}$ identified that the risk of hospitalization of patients undergoing family intervention was lowered by $40 \%$. Psychotic symptoms significantly reduced at 12 months, rather than at 18 months. At 12 months and 18 months, social impairment in the family intervention group was dramatically diminished. and conformity increased by the introduction of practical role-playing and exercises on coping skills and problem-solving strategies by participants and their families.

The third study was done by Ran et al ${ }^{33}$ showed that psycho-educational family intervention can still be effectual in 14-year follow-up, particularly in adherence with therapy and patients social functioning. In addition, the family psycho-educational intervention group showed a slightly higher incidence of antipsychotic treatment and a higher workability rate than the other two groups. Marvin et al ${ }^{26}$ published the fourth study, which found that FFT-CHR concentrated more on teamwork and problem-solving skills training than EC. In addition, FFT-CHR provides a three-module module that focuses on the ability of patients and their families to cope with psychotic symptoms. The last study was carried out by Niksalehi et al ${ }^{27}$. The results showed that family psycho-education can have a major impact on the risk of readmission and the period of hospital stay.

Seventhly, a study undertaken by Eisner et al ${ }^{28}$ guarantees that the study culminated in three major themes: firstly, recognizing relapse risk factors; secondly, early signs detection; thirdly, reacting properly to deterioration. Finally, the Fikreyesus et al 29 study reported several findings: first, psychotic relapse in family patients was $72 \%$ less than in patients living alone; second, psychotic relapse in patients who comply with the treatment was $69 \%$ lower than in patients that refused to comply; third, in patients with high social support levels, the development of psychotic relapse was $48 \%$ lower than in patients without social support; last, in those who indicated religious support, the psychotic relapse was $45 \%$ lower than those in patients without religious support.

\section{Discussion}

\section{Summary of evidence}

Schizophrenia patients usually have a regular familial touch. The family should also be interested in treating and caring for their patient as much as possible, so it can acknowledge the rehabilitation of that patient and meet the need of the family to seek knowledge, care, and treatment. Thus, different relapse prevention intervention strategies emphasize the ability of the patient and their families' interrelationship to minimize or prevent psychotic symptoms. The literature reviewed for this study apparently illustrates the extent of the work carried out in this field. Much study has highlighted the main approaches of the reciprocal intervention of preventive patients and families of psychotic relapse. However, research focused mostly on developing approaches to relapse prevention. Efforts also propose and test the policy efficacy and effect of those interventions on the well-being of psychotic patients to prevent psychotic symptoms. 
The study has shown the benefits of psychosocial therapy by preparing patients and families with various psychosocial intervention methods in both clinical and societal aspects ${ }^{34}$. The other study proves the Cognitive remediation capability to reduce such psychotic symptomatic and reduce the number of deficits that combined with health benefits to the Multi-family psycho-education group (MFG) ${ }^{2}$. A further study revealed the Efficient preparation of patients and their families to utilize and cope with Integrated Psychological Intervention (IPI) and incorporation of patients with the other intervention strategies such as cognitive-behavioral intervention, and cognitive remediation, multifamily psycho-education, and group skills training, lead to reduction the early prodromal psychotic state (EIPS) ${ }^{21}$.

One Study has shown that the second episode of psychosis can be stopped with new psycho-social therapy for seven months. Together, this research will change the handling of and prevention of psychotic relapses, with emphasis on both patients and their families. However, relapse rates were lower and incidence was delayed at the completion of treatment (7 months) and 12 months with the RPT group contrast with the traditional treatment. However, at 18, 24, and 30 months, these disparities were not sustained ${ }^{22}$. in addition, one study focusing on integrated the technology with relapse prevention by creat ITAREPS as a relapse prevention program. The effectiveness of ITAREPS for the prevention of relapses was high and we reported that ITAREPS is a successful tool during the early stages of relapses through the identification of symptoms of relapse and the increase of dosage during the warning state by using EWSQ (early warning sign questionnaires) through phone calls weekly for both patients and their families to detect the early warning signs. thus, the ability of patients and their families to monitor and prevent psychotic symptom is effective $^{23}$.

Over a third of the studies $(n=5,42 \%)$ reported that improving outcomes including lower risk of relapse, and decreased severity of symptoms as well as improved medication compliance, is a consequence of the efficacy and effectiveness of family intervention programs. Moreover, family-focused intervention and culturally awareness aspects have moderately high and significant beneficial impacts on psychotic symptoms ${ }^{24}$. Furthermore, applying constructive roleplaying and activities to coping skills and problemsolving strategies by both patients and their families decreases hospitalization complications and increases the social functioning and condition of mental health for Schizophrenic patients ${ }^{32}$. In addition, motivation for improved medication adherence is a highly important aim for a psycho-educational family intervention in environments where family members have actively engaged in patient care, suggesting a link between the risk of relapse and non-adherence ${ }^{33}$. As well, familyfocused therapy for patients at clinical high risk for relapse including three-module; all of these modules focusing the ability of patients and their families to deal with psychotic symptoms ${ }^{26}$. Thus, family education will strengthen their effective interactions with patients, but it can also be seen as one of the biggest obstacles in the implementation of preparatory service for severe disease patients and has to be managed and evaluated in a continuous phase ${ }^{27}$.

As the qualitative study found during a deep, participants spoke very reflectively about their experiences before a relapse. Pre-relapse symptoms, the degree to which they could recall them and their answers to them is significantly different from those of the main participants. A successful supporting source was therefore an allocated family or friend, although it was not always possible to support important others ${ }^{28}$. The last study found that patient-family relationships, religious support, high level of social support, and drug adherence were independently related to a decreased risk of psychotic relapse ${ }^{29}$.

\section{Implications for future practice, policy, and research}

The results of this review highlight the importance of reporting the ability of the patients and their families to minimize and preventing psychotic relapse by using different relapse prevention intervention approaches. Various relapse prevention intervention approaches are recommended in clinical practice for psychotic patients. This would help clinicians, nurses, and families to practice effectively to reduce the relapse rate among patients suffering from schizophrenia. So that, 
these recommendations and needs pull the clinician and psychiatric nurse to do massive comprehensive researches to offering preliminary evidence that supports practice about the implementation of relapse prevention intervention and affect future research.

\section{Conclusions}

Many patients diagnosed with schizophrenia have family support. The role of the family as partners in treatment has the ability to increase the recovery of the patient and resolve the need for awareness, help, and improved expertise of the family for its care. In this sense, there is clear proof that patients and their families benefit from involvement in reciprocal preventive programs. This review gives an overview of relapse prevention intervention approaches to reduce the readmission rate, prevent psychotic symptoms, and sustain remission by incorporating families to support patients with psychotic relapse. Our review revealed that many strategies based on evidence indicate the significance of family intervention in therapy and emphasize the importance of effective cooperation with patients. Moreover, the outcomes have been impacted by improved treatment strategies over the last decade, including different approaches of relapse prevention intervention such as more effective psychosocial approaches, family-focused therapy, and family psycho-education therapy that have been developed to help patients and their families to prevent psychotic symptoms. Therefore, the relapse prevention interventions help to improve awareness and commitment to schizophrenia patients' care by families. On the other hand, relapse prevention interventions are not commonly available and it is a vital but not enough step to take learning practitioners to this technique. It is becoming increasingly evident that structural and multilevel approaches to transform practice with a strong focus on corporate help are required. Future innovations to strengthen family engagement in the implementation of care include studies to improve families' engagement, including through less costly approaches and improved awareness of how and to whom relapse prevention offers benefits

Ethical Statement: Ethical permissions were not necessary
Funding statement: No particular grant has been given to this research by any public, private or non-profit funding organization.

Conflict of Interest Statement: No conflicts of interest.

\section{References}

1. World health orgnization. key fact sheet,2019. https://www.who.int/news-room/fact-sheets/detail/ schizophrenia.

2. Fusar-Poli, P., McGorry, P. D., \& Kane, J. M. Improving outcomes of first-episode psychosis: an overview. World psychiatry. 2017; 16(3), 251-265.

3. Grover, S., Pradyumna, \& Chakrabarti, S. Coping among the caregivers of patients with schizophrenia. Industrial psychiatry journal. 2015; 24(1), 5-11. https://doi.org/10.4103/09726748.160907.

4. National Institute of Mental Health. schizophrenia. 2018 https://www.nimh.nih.gov/health/statistics/ schizophrenia.shtml.

5. Olivares, J. M., Sermon, J., Hemels, M., \& Schreiner, A. Definitions and drivers of relapse in patients with schizophrenia : a systematic literature review.2013; Annals of General Psychiatry. 2013; 1-11. https://doi.org/10.1186/1744-859X-12-32.

6. Orygen. Preventing relapse in first episode psychosis. 2016; 6. Retrieved from http://www. orygen.org.au.

7. Witkiewitz, K. Preventing Relapse. Psychologists' Desk Reference. 2015; 350-354. https://doi. org/10.1093/med:psych/9780199845491.003.0073

8. Gross, S. Relapse Prevention. Psych Central. 2020; Retrieved on October 28, 2020, from https:// psychcentral.com/lib/relapse-prevention/

9. Al Ja'afreh, S., Allari, R., \& Hamdan-Mansour, A. Gender differences in life satisfaction: The moderation role of social support among older persons. Medico-Legal Update, 2021; 21 (1), 935941.

10. Aldiqs, M. \& Hamdan-Mansour, A. The role of religion and spirituality in alcohol use treatment and recovery: An integrative review. Medico-Legal Update. 2021; 27 (1), 599-605. 
11. Hamdan-Mansour, A. \& Bandak, A., Puskar, K. Effectiveness of Cognitive-Behavioral Intervention on Depressive Symptomatology, Stress and Coping Strategies among university students in Jordan. Issues in Mental Health Nursing. 2009; 30, 188196.

12. Hamdan-Mansour, A., Constantino, R., Shishani, K., Safadi, R. \& Bani-Mustafa, R. Evaluating psychological and mental consequences of abuse among abused Jordanian women. Eastern Mediterranean Health Journal.2012; 18 (3), 205212.

13. Al Abaiat, D., Hamdan-Mansour, A., Hanouneh, S., \& Ghannam, B. (2016). Psychosocial predictors of relapse among alcohol dependents in Jordan. Current Drug Abuse Reviews. 9 (1), 19-25.

14. Hamaideh, S., Hamdan-Mansour, A., \& Al Hamdan, A. Levels and correlates of disability in life skills among patients with schizophrenia. Current Psychiatry Research and Reviews. 2020; 16(4), 275282. DOI: $10.2174 / 2666082216999200905133918$

15. Issa, E., Hamdan-Mansour, A., \& Hamaideh, S. Knowledge of and adherence to antipsychotic medications among individuals diagnosed with schizophrenia. Jordan Medical Journal. 2019; 53 (3), 113-126.

16. Keepers, G. A., Fochtmann, L. J., Anzia, J. M., Benjamin, S., Lyness, J. M., Mojtabai, R., ... \& Young, A. S. The American psychiatric association practice guideline for the treatment of patients with schizophrenia. American Journal of Psychiatry. 2020; 177(9), 868-872.

17. Critical Appraisal Skills Programme. CASP qualitative checklist, 2018.

18. Thomas, BH, Ciliska, D, Dobbins, M, \& Micucci, S. Quality assessment tool for quantitative studies dictionary: the Effective Public Health Practice Project (EPHPP). McMaster University.2008.

19. Critical Appraisal Skills Programme. CASP qualitative checklist, 2018

20. Sungur, M., Soygür, H., Güner, P., Üstün, B., Cetin, I., \& Falloon, I. R. Identifying an optimal treatment for schizophrenia: A 2-year randomized controlled trial comparing integrated care to a highquality routine treatment. International Journal of
Psychiatry in Clinical Practice. 2011; 15(2), 118127.

21. Bechdolf, A., Wagner, M., Ruhrmann, S., Harrigan, S., Putzfeld, V., Pukrop, R., \& Klosterkötter, J. Preventing progression to first-episode psychosis in early initial prodromal states. The British Journal of Psychiatry. 2012; 200(1), 22-29.

22. Gleeson, J. F., Cotton, S. M., Alvarez-Jimenez, M., Wade, D., Gee, D., Crisp, K., \& McGorry, P. D. A randomized controlled trial of relapse prevention therapy for first-episode psychosis patients: outcome at 30-month follow-up. Schizophrenia Bulletin. 2013; 39(2), 436-448

23. Komatsu, H., Sekine, Y., Okamura, N., Kanahara, N., Okita, K., Matsubara, S., ... \& Iyo, M. Effectiveness of Information Technology Aided Relapse Prevention Programme in Schizophrenia excluding the effect of user adherence: a randomized controlled trial. Schizophrenia research. 2013; 150(1), 240-244.

24. Weisman de Mamani, A., Weintraub, M. J., Gurak, K., \& Maura, J. A randomized clinical trial to test the efficacy of a family-focused, culturally informed therapy for schizophrenia. Journal of Family Psychology. 2014; 28(6), 800

25. Thomas BH, Ciliska D, Dobbins M, Micucci S. Dictionary for Quality Assessment Tool for Quantitative Studies (EPHPP). 1998; https:// merst.ca/wp-content/uploads/2018/02/qualiltyassessment-dictionary_2017.pdf

26. Marvin, S. E., Miklowitz, D. J., O’Brien, M. P., \& Cannon, T. D. Family-focused therapy for individuals at clinical high risk for psychosis: treatment fidelity within a multisite randomized trial. Early intervention in psychiatry. 2016; 10(2), 137-143.

27. Niksalehi, S., Namazi, S., Tashk, M., Bavaghar, S., Afandi, M., \& Jamalizadeh, F. Impact of Family Psychoeducation Intervention on Relapse Prevention in Hospitalized Psychiatric Patients. 2019; Hormozgan Medical Journal, 23(4).

28. Eisner, E., Barrowclough, C., Lobban, F., \& Drake, R. Qualitative investigation of targets for and barriers to interventions to prevent psychosis relapse. BMC psychiatry. 2014; 14(1), 1-14. 
29. Fikreyesus, M., Soboka, M., \& Feyissa, G. T. Psychotic relapse and associated factors among patients attending health services in Southwest Ethiopia: a cross-sectional study. BMC psychiatry. 2016; 16(1), 1-10.

30. Sungur, M., Soygür, H., Güner, P., Üstün, B., Cetin, I., \& Falloon, I. R. Identifying an optimal treatment for schizophrenia: A 2-year randomized controlled trial comparing integrated care to a highquality routine treatment. International Journal of Psychiatry in Clinical Practice. 2011; 15(2), 118127 .

31. Breitborde, N. J., Moreno, F. A., Mai-Dixon, N., Peterson, R., Durst, L., Bernstein, B., \& McFarlane, W. R. Multifamily group psychoeducation and cognitive remediation for first-episode psychosis: a randomized controlled trial. BMC psychiatry. 2011; 11(1), 1-7.

32. Mayoral, F., Berrozpe, A., de la Higuera, J.,
Martinez-Jambrina, J. J., de Dios Luna, J., \& TorresGonzalez, F. Efficacy of a family intervention programme for prevention of hospitalisation in patients with schizophrenia. A naturalistic multicenter controlled and randomised study in Spain. Revista de Psiquiatría y Salud Mental (English Edition). 2015; 8(2), 83-91.

33. Ran, M. S., Chan, C. L., Ng, S. M., Guo, L. T., \& Xiang, M.Z. The effectiveness of psychoeducational family intervention for patients with schizophrenia in a 14-year follow-up study in a Chinese rural area. Psychological medicine. 2015; 45(10), 2197.

34. Sungur, M., Soygür, H., Güner, P., Üstün, B., Cetin, I., \& Falloon, I. R. Identifying an optimal treatment for schizophrenia: A 2-year randomized controlled trial comparing integrated care to a highquality routine treatment. International Journal of Psychiatry in Clinical Practice. 2011; 15(2), 118127. 